sequence type, strongly suggesting that the early MSSA examined here represented the progeny of a strain that served as one of the first $S$ aureus recipients of the methicillin-resistance determinant in Europe. The genetic background of this group of early MSSA isolates was also very similar to that of the widely disseminated contemporary "Iberian clone" of MRSA, suggesting that genetic determinants present in early MSSA and essential for some aspects of the epidemicity or virulence of these strains, may have been retained by this highly successful contemporary MRSA lineage.

FROM: Crisostomo MI, Westh H, Tomasz A, Chung M, Oliveira DC, de Lencastre. The evolution of methicillin resistance in Staphylococcus aureus: similarity of genetic backgrounds in historically early methicillin-susceptible and -resistant isolates and contemporary epidemic clones. Proc Natl Acad Sci U S A 2001;98:9865-9870.

\section{Detection of Nosocomial Tuberculosis With Molecular Typing}

Tudo and coinvestigators from Barcelona, Spain, conducted a study to investigate the use of restriction fragment-length polymorphism to detect unsuspected cases of nosocomial transmission of TB among patients who had been admitted to a university hospital. One hundred fifty-one samples of Mycobacterium tuberculosis isolated from patients with pulmonary TB were studied. The isolates from 37 patients (24.5\%) defined 11 clusters. None of the patients infected with these cluster isolates had hospital stays that coincided with one another, and, for $5.4 \%$ of the patients, the epidemiological link clearly was outside the hospital. Previous incarceration was associated with infection with cluster isolates. In addition, 109 patients without TB (41 of whom were infected with HIV) who shared a room with patients who had TB were followed for 18 to 60 months. Among the patients who survived, secondary cases of TB due to nosocomial transmission were not detected.

FROM: Tudo G, Gonzalez J, Gatell JM, Cayla JA, Martinez E, Garcia A, et al. Detection of unsuspected cases of nosocomial transmission of tuberculosis by use of a molecular typing method. Clin Infect Dis 2001;33:453-459.

\section{Nasal Carriage of $S$ aureus: Risk Factor for Skin Infections}

Toshkova and coinvestigators have reported on a study designed to investigate the significance and the relation between nasal carriage of Staphylococcus aureus and staphylococcal skin infections. Thirty-one $S$ aureus strains, isolated from 12 patients with chronic and recurrent skin infections, one patient with septicemia, and one patient with otitis externa were studied. The staphylococcal strains were isolated from the site of infection and from the anteri- or nares of each patient. The identity of both strains of each pair could be demonstrated by determination of phenotypic properties and by genotyping of the isolates. The phenotypic properties included hemolytic activities, antibiotic resistance data, and the production of enterotoxins. The identity was additionally confirmed by phage typing, by determination of the size and the number of repeats of the X region of $s p a$ gene, by determination of gene polymorphisms of coa gene, and by macrorestriction analysis of the chromosomal DNA of the isolates by pulsed-field gel electrophoresis.

The results showed an identity of the $S$ aureus obtained from anterior nares and from skin infection of each patient, indicating the importance of nasal carriage of these bacteria for development of human skin infection.

FROM: Toshkova $\mathrm{K}$, Annemuller $\mathrm{C}$, Akineden $O$, Lammler $C$. The significance of nasal carriage of Staphylococcus aureus as risk factor for human skin infections. FEMS Microbiol Lett 2001;202:17-24.

\section{Attitudes Toward Infection Control Programs in Australia}

Debate remains over the core activities of infection control (IC) programs. Differences in stakeholder opinions must be considered if consensus panel guidelines and recommendations are to be applied broadly. Murphy and McLaws have reported on a survey of administrators and clinicians employed in hospitals in New South Wales, Australia. Respondents self-reported their levels of agreement with affirmative statements regarding the role of the infection control practitioner (ICP) and the essential requirements and infrastructure of IC programs. The study population included administrators and clinicians in each public, private, and freestanding day hospital in New South Wales. Respondents reported the intensity of their agreement with 16 affirmative statements relating to IC program infrastructure and resources and the ICP's role and responsibilities.

The overall response rate was $62.1 \%$ (587/945). Clinicians $(349 / 587)$ and administrators (238/587) accounted for $59.5 \%$ and $40.5 \%$ of the response rate, respectively. Overall, administrators and clinicians reported greatest levels of agreement for those elements not requiring additional resources.

The authors concluded that the extent of divergence between administrators and clinicians is not so great that it cannot be resolved. They advocate better communication between clinicians and administrators in conjunction with objective strategic planning and offer their findings as a guide for ICPs to either establish or negotiate the core components of their IC program.

FROM: Murphy CL, McLaws Ml. Variation in administrators' and clinicians' attitudes toward critical elements of an infection control program and the role of the infection control practitioner in New South Wales, Australia. Am J Infect Control 2001;29:262-270. 